\title{
L'Autunno caldo: \\ lotte operaie, sindacato e mutamento sociale
}

\author{
Fabrizio Loreto* e Stefano Musso**
}

The "Hot Autumn": workers' struggles, trade unions and social change

1969 is a key year in the history of modern Italy, characterised by an impressive wave of radical struggles and worker mobilisation. This piece analyses the causes of this cycle of conflict, which were created by the contradictions inherent in the industrial 'boom', as well as the tensions which had built up in the industrial relations system. Those sectors of the trade unions which were most receptive to worker demands for profound change were among the protagonists of the 1968-1969 period. The workers' struggles had a great influence on Italian society in the 1970s and made a defining contribution to the reforms in civil and social rights.

Key words: "Hot Autumn", Industrial relations, Trade Unions, Workers' rights Parole chiave: "Autunno caldo", Relazioni industriali, Sindacato, Diritti dei lavoratori

L'Autunno caldo del 1969, caratterizzato da lotte operaie radicali che determinarono rilevanti cambiamenti economici e giuridici nel mondo del lavoro e nella società, ha rappresentato senza dubbio uno dei passaggi più importanti della storia dell'Italia repubblicana ${ }^{1}$. L'irrompere e il dilagare delle mobilitazioni operaie catalizzò spinte al cambiamento emerse negli anni precedenti, in una società capace di modernizzare l'apparato produttivo e liberare risorse economiche, ma ancorata a forme di disciplinamento sociale tradizionali e, dal punto di vista dei diritti civili, piuttosto arretrate. Gli effetti, per molti aspetti, furono duraturi. Riteniamo dunque utile ripercorrere gli antefatti del 1969, gli sviluppi che ne seguirono, per tratteggiare infine alcuni cambiamenti della società italiana che, nel crogiuolo di quel periodo, ebbero il loro brodo di coltura.

* Dipartimento di studi storici, via S. Ottavio 20, 10124 Torino; fabrizio.loreto@unito.it

** Dipartimento di studi storici, via S. Ottavio 20, 10124 Torino; stefano.musso@unito.it

${ }^{1}$ Il primo paragrafo di questo intervento è scritto da Stefano Musso, il secondo da Fabrizio Loreto; la parte introduttiva e il terzo paragrafo sono frutto di una stesura comune. Cfr. D. Giachetti, L’Autunno caldo, Ediesse, Roma 2013. 
L'Autunno caldo si colloca al centro di un lungo ciclo di agitazioni che aveva preso avvio nel 1960 e si sarebbe concluso con quello che si potrebbe chiamare 1" "Autunno freddo" del 1980, con la sconfitta dei lavoratori e del sindacato nella vertenza dei "35 giorni" alla Fiat. È indubbio che proprio il sindacato fu uno dei soggetti più importanti degli anni '60 e ' 70 , nonché il principale protagonista del "lungo 1969". Nel 2017, in un editoriale su questa rivista, Pietro Causarano si interrogava sulla distanza notevole che separa la fase alta vissuta dalle confederazioni nella stagione della supplenza sindacale e un tempo presente segnato dalla «normalità di una crisi» che sembra «senza fine»². L'autore individuava proprio nel carattere ambivalente del sindacato, insieme «movimento sociale» e «soggetto istituzionale», la chiave interpretativa per spiegare «la fatica di Sisifo» cui era perennemente costretto: obbligato a rincorrere nel breve periodo le continue trasformazioni del mondo del lavoro, ma anche capace di rappresentare, nel lungo periodo, un elemento essenziale di stabilità democratica. Tale ambivalenza, presente sin dalle origini, risulta evidente tanto nei periodi di arretramento, quanto nei momenti di progresso: tra questi, l'Autunno caldo fu certamente il più esaltante.

\section{Alle origini dell'Autunno caldo}

Il 1960 segnò la fine della debolezza sindacale degli anni '50, dovuta agli errori strategici del centralismo contrattuale della Cgil e all'azione repressiva del padronato, nel quadro della divisione ideologica generata dai riflessi interni della guerra fredda. Nei primi anni '60 la ripresa delle agitazioni fu favorita dalla piena occupazione al Nord - un cambiamento secolare del mercato del lavoro, finalmente non più squilibrato a favore della domanda e dal riavvicinamento tra i sindacati, in particolare tra la Cgil e la Cisl. La prima abbandonò il centralismo accettando la prospettiva della contrattazione aziendale, mentre la seconda vide una radicalizzazione delle sue categorie industriali in seguito al rifiuto padronale del modello di relazioni collaborative incentrato sulla strategia della produttività ${ }^{3}$.

I rinnovi contrattuali dei primi anni '60 ottennero la contrattazione articolata e aziendale - limitata di fatto a premi di produzione - aumenti salariali cui seguì il recupero sui prezzi, con una tendenza inflattiva e un deficit della bilancia dei pagamenti, cui le autorità monetarie reagirono con la stretta creditizia, causando la congiuntura negativa del 1964-65. Quest'ultima si river-

${ }^{2}$ P. Causarano, La fatica di Sisifo del sindacato, «Passato e presente», 35 (2017), n. 100, pp. 9-24.

${ }^{3}$ F. Loreto, Il sindacalismo nell'Italia repubblicana: organizzazioni, politiche, culture, «democrazia e diritto», 2013, n. 3-4, pp. 171-212; P. Trionfini, La laicità della Cisl. Autonomia e unità sindacale negli anni Sessanta, Morcelliana, Brescia 2014. 
berò sui rinnovi contrattuali del 1966, firmati al ribasso: gli industriali furono lieti delle magre concessioni fatte, ma la ripresa della crescita economica avrebbe ben presto fomentato di nuovo la conflittualità operaia. Questa riprese vigore nel 1968, contestualmente alla protesta studentesca, in particolare con le vertenze aziendali su cottimi e premi, e con le battaglie generali sulle pensioni e contro le "gabbie salariali", cui si aggiunsero gli scioperi per gli eccidi di Avola e Battipaglia.

Lo scoppio, spesso spontaneo, degli scioperi tra il '68 e il '69 arrivò all'apice del fordismo e ne costituì certamente uno dei fattori di crisi negli anni successivi. Le agitazioni ebbero una partecipazione di massa che vide protagonisti da un lato i vecchi militanti, operai qualificati, che vivevano le nuove lotte come una rivincita sugli anni duri della repressione, dall'altro gli operai comuni, immigrati dalle campagne del Nord o dal Mezzogiorno, la cui carica rivendicativa derivava dalla miscela esplosiva tra pesantezza delle condizioni di lavoro in fabbrica e disagi per la carenza di abitazioni, servizi sanitari, trasporti, edifici scolastici, nei centri urbani investiti dall'aumento troppo rapido della popolazione, per non dire delle difficoltà dovute alle barriere linguistiche e alle ghettizzazioni (dal "non si affitta ai meridionali" alle classi differenziali per i figli a scuola). Il contesto della conflittualità radicale fu quello di un mutamento socio-culturale profondo e concentrato nel tempo, con una massa di circa quattro milioni di lavoratori in movimento, in un paese che in un quindicennio aveva vissuto la trasformazione epocale, passando da agricolo a industriale ${ }^{4}$.

Alla fine del lungo miracolo economico i redditi delle famiglie operaie erano senza dubbio migliorati, ma più per l'aumento degli occupati che per l'incremento dei salari individuali. La produttività del lavoro, e con essa i profitti, erano aumentati più dei salari. Il tenore di vita era cresciuto; però molti lavoratori, specie i giovani immigrati, erano trattenuti sulla soglia del pieno accesso a una società del benessere i cui consumi, ormai da anni, erano oggetto di campagne pubblicitarie pervasive. Si diffusero così istanze acquisitive che si riverberarono nelle richieste di consistenti aumenti salariali. Le rivendicazioni operaie godettero, in un primo tempo almeno, di una opinione pubblica in buona parte favorevole, a causa della diffusa convinzione che i lavoratori avessero ricevuto meno del contributo da loro offerto allo sviluppo economico. L'Autunno caldo, dunque, fu anche il prodotto delle contraddizioni dello sviluppo.

Sul terreno specifico delle relazioni industriali, a fine anni '50, con il distacco delle imprese di stato dalla Confindustria e la costituzione dell'Intersind per l'Iri e dell'Asap per l'Eni, si era aperto una sorta di doppio canale

${ }^{4} \mathrm{Nel} 1951$ l'agricoltura contava il 42,2\% della popolazione attiva, mentre nel 1971 la quota dell'industria arrivò al 44,4\%. Cfr. S. Gallo, Senza attraversare le frontiere. Le migrazioni interne dall'Unità a oggi, Laterza, Roma-Bari 2012. 
nel sistema contrattuale. Le relazioni di lavoro distinte che si avviarono nel campo delle partecipazioni statali avrebbero forse potuto smussare il tasso di conflittualità molto elevato che si sarebbe manifestato nel 1968-69. A partire dal 1962, infatti, i sindacati firmarono con Intersind e Asap protocolli che implicavano il pieno riconoscimento del ruolo del sindacato e introducevano clausole di tregua.

Il contratto dei metalmeccanici del 1966 non mise mano alla rappresentanza aziendale. Venne infatti rinnovato l'accordo interconfederale del 1953 sulle commissioni interne lasciando il sistema sostanzialmente intatto, ignorando che negli stabilimenti più grandi i pochi membri della commissione interna non erano più in grado di raccogliere e trattare le problematiche dei lavoratori a causa del rapporto numerico inadeguato tra i pochi commissari e la massa dei rappresentati, e delle persistenti limitazioni all'esercizio dei diritti sindacali. Le direzioni aziendali continuavano a stringere i lacci della disciplina produttiva per cogliere le occasioni offerte dai mercati in espansione, nell'illusione che le tensioni sociali potessero essere sciolte dallo sviluppo, non percependo l'opportunità di una rappresentanza capace di portare alla luce il fuoco che covava sotto la cenere.

Nel 1966, tuttavia, nel quadro del riformismo del primo centro-sinistra, si ebbe l'approvazione della legge 604, che introdusse la giusta causa nei licenziamenti individuali, anticipando in qualche misura lo Statuto dei diritti dei lavoratori del 1970. Il 1967 fu un anno di passaggio. Ripartirono gli investimenti, le migrazioni e le vertenze aziendali, incentrate prevalentemente su cottimi e premi, mentre iniziò ad affacciarsi la tematica della tutela della salute operaia. Comparvero anche i primi gruppi di studenti ai cancelli delle fabbriche.

La Confindustria tardò ad accogliere l'idea di un modello di relazioni industriali basato sul riconoscimento del ruolo del sindacato nella gestione dei rapporti di lavoro. A lungo le sue posizioni restarono ancorate agli schemi degli anni '50. Questo ritardo sarebbe stato colmato solo con la Commissione Pirelli, la cui relazione fu approvata nel gennaio 1970, quando la conflittualità era ormai dilagata ed era impossibile incanalarla in un quadro normativo condiviso. Infatti, lo stesso destino toccò anche al modello partecipativo di Intersind e Asap. Giuseppe Glisenti, presidente dell'Intersind, nella relazione all'assemblea annuale del 1969 sostenne ancora che bisognava tutelare l'azione del sindacato, un elemento fondamentale della regolazione contrattuale; l'anno successivo, però, si rammaricò che la contrattazione non realizzasse più l'introduzione di norme valide per il periodo concordato, e che gli accordi fossero divenuti un gradino dal quale muovere, prima della scadenza, per avanzare ulteriori rivendicazioni, in una sorta di contrattazione permanente ${ }^{5}$.

${ }^{5} \mathrm{G}$. Berta, L'Italia delle fabbriche. Genealogie ed esperienze dell'industrialismo nel Novecento, il Mulino, Bologna 2001, pp. 188-204. 
Le tensioni generate da quella che può essere considerata una vera e propria "crisi di sviluppo", avevano creato una situazione in cui non era sufficiente proporre una strategia di gestione partecipata quale quella tentata nelle imprese di stato.

\section{Gli sviluppi del "lungo 1969": il protagonismo dei lavoratori e del sindacato}

La rottura provocata dal movimento del '68 modificò consolidati equilibri politici e sociali in diversi sistemi nazionali, anche molto diversi tra loro $^{6}$. In Italia, tra la fine del '68 e l'inizio del '69 la gran parte dei lavoratori partecipò in modo attivo al processo di rinnovamento in atto nel sindacato, considerato il soggetto capace di assicurare uno sbocco positivo alle mobilitazioni, attraverso la conquista di novità importanti sul piano salariale e normativo. Per valutare appieno il significato storico del '69 occorre spiegare l'ampiezza e la radicalità delle lotte operaie, che non avevano precedenti nella storia nazionale e non avrebbero più raggiunto quei livelli di intensità negli anni successivi.

Il volume di Giuseppe Maione 1969. L'autunno operaio aiuta a chiarire alcuni aspetti fondamentali, ben noti agli storici del lavoro, per capire la dimensione quantitativa e il valore qualitativo di quelle lotte ${ }^{7}$. È vero, infatti, che sul terreno strettamente economico la stagione del boom aveva prodotto una situazione di piena occupazione soprattutto nelle zone più industrializzate e urbanizzate del Centro-Nord, che la congiuntura negativa del 1964-65 aveva solo in minima parte ridimensionato. Una così estesa quantità di manodopera al lavoro, mai registrata nel paese, accompagnata da una larga disponibilità di occasioni d'impiego, rappresentava senza dubbio un'importante arma a disposizione del movimento operaio. È anche vero che negli anni '60, dopo la drammatica avventura del governo Tambroni, la novità del centrosinistra aveva rasserenato in parte il clima politico, con effetti positivi anche sul terreno delle relazioni industriali e dei rapporti tra i sindacati stessi, dopo gli eccessi di ostilità vissuti nel periodo più buio della guerra fredda. Sul piano culturale, poi, il percorso di rinnovamento ideale e progettuale che investì trasversalmente tanto il mondo cristiano (dalla vicenda conciliare all'esperienza delle Acli, fino alle manifestazioni più radicali del dissenso cattolico) quanto il variegato universo delle sinistre socialiste e comuniste (animato soprattutto dall'operazione di revisione del marxismo in chiave operaista), si rivelò

${ }^{6}$ M. Flores-G. Gozzini, 1968. Un anno spartiacque, il Mulino, Bologna 2018.

${ }^{7}$ G. Maione, 1969. L'autunno operaio, manifestolibri, Roma 2019. Cfr. P. Causarano, La fabbrica fordista e il conflitto industriale, in Storia del lavoro in Italia, diretta da F. Fabbri, v. II, Il Novecento, 1945-2000: la ricostruzione, il miracolo economico, la globalizzazione, a cura di S. Musso, Castelvecchi, Roma 2015, pp. 59-101. 
particolarmente efficace. Da ultimo, è quasi scontato sottolineare che, senza il movimento del '68 studentesco, senza cioè quella contestazione giovanile così radicale e irriverente, a tratti irruenta, che dagli atenei e dalle scuole si trasferì presto nella società, trovando negli operai delle fabbriche il primo e più naturale interlocutore, il '69 operaio non avrebbe avuto di certo la stessa carica dirompente di ribellione.

Tuttavia, l'elemento "chiave", la causa che più di tutte illustra la portata storica dell'onda conflittuale del 1969 e la novità delle forme di lotta e dei contenuti rivendicativi, nonché la diffusione capillare (di certo non confinabile - come, per la verità, rischia di fare Maione - ai casi della Fiat di Torino, della Pirelli di Milano e della Montedison di Marghera), sta nella durezza dei meccanismi del taylorismo, cioè dell'organizzazione scientifica della produzione: considerata dalle imprese "oggettiva" e dunque una sorta di tabù immodificabile (in quanto appunto scientifica, espressione del massimo di razionalità). Nella realtà concreta delle fabbriche tale organizzazione impose ritmi e metodi di lavoro spesso brutali, produsse ambienti malsani, causò frequenti infortuni e numerose malattie, logorò la salute fisica e psicologica di molti lavoratori ${ }^{8}$.

Dall'indagine storica emergono i tanti e diffusi casi aziendali segnati dalla logorante nocività ambientale e da rigide gerarchie organizzative, che colpivano soprattutto la manodopera più dequalificata. Fu contro tale condizione che si scatenò l'insubordinazione operaia. I conflitti di lavoro dilagarono pressoché ovunque, dalle carrozzerie di Mirafiori, epicentro dello scontro di classe nella più grande fabbrica del paese, alle medie imprese della "Terza Italia", dal "triangolo industriale" alle "cattedrali nel deserto" del Meridione, dall'industria manifatturiera all'edilizia, nel mondo dei trasporti, nel privato come nel pubblico, dentro gli ospedali, nelle scuole 9 . In tale situazione, piuttosto caotica ma segnata dalla tendenza comune a contrastare ogni forma di gestione autoritaria del potere nei luoghi di lavoro, si realizzò quella sorta di rivoluzione copernicana che, all'oggettività di regole fissate quasi sempre unilateralmente dalle direzioni aziendali, cercò di sostituire la soggettività operaia, cioè il punto di vista dei lavoratori dipendenti, i loro bisogni più elementari, le loro aspirazioni a un lavoro decente e a una vita dignitosa.

Per comprendere l'esito positivo di quella sorprendente stagione di mobilitazione popolare occorre soffermarsi sul ruolo essenziale giocato dal sindacato $^{10}$. Con due precisazioni. Anche il mondo sindacale, specie nella fase ini-

${ }^{8}$ G. Maifreda, La disciplina del lavoro. Operai, macchine e fabbriche nella storia italiana, Bruno Mondadori, Milano 2007, pp. 282-300.

${ }_{9}$ S. Bartolini-P. Causarano-S. Gallo (a cura di), Un altro 1969: i territori del conflitto in Italia, Edizioni Ndf, Palermo 2020.

${ }^{10}$ P. Causarano-L. Falossi-P. Giovannini (a cura di), Il 1969 e dintorni. Analisi, riflessioni e giudizi a quarant'anni dall' "Autunno caldo", Ediesse, Roma 2010; A. Ciampani-G. Pellegrini (a cura di), L'autunno sindacale del 1969, Rubbettino, Soveria Mannelli 2013. 
ziale della rivolta sessantottina, e per un periodo non breve, fu oggetto della contestazione giovanile, inserito in un unico grande calderone che comprendeva scuole e università, forze dell'ordine e magistratura, chiesa e famiglie, governo e partiti, tutti accomunati dall'accusa di perpetuare i medesimi meccanismi autoritari che rendevano soffocante il "sistema"". La contestazione, però, si rivelò salutare. In seguito, infatti, i sindacati riuscirono faticosamente a innescare un processo autonomo di trasformazione interna, che dapprima li allontanò dagli strali più acuminati della contestazione $\mathrm{e}$, in un secondo momento, li mutò in veri e propri soggetti della contestazione: i soli in grado di confrontarsi, anche in modo aspro, con le accuse provenienti dal basso, dalla stessa base, riuscendo in un'opera di contaminazione con ampi settori del mondo del lavoro che garantì loro, infine, di governare l'intero movimento dei lavoratori, indirizzandolo verso uno sbocco certamente di natura contrattuale, ma anche pienamente politico. Tale processo di «sindacalizzazione della contestazione», come venne definito in maniera mirabile da Gino Giugni all'inizio degli anni '70, durò alcuni mesi, tra il 1968 e il 1969, precedendo l'Autunno caldo e culminando con esso ${ }^{12}$.

Tuttavia - ed è questa la seconda precisazione - la complessa opera di sindacalizzazione della contestazione non coinvolse l'intero sindacato, ma solo una parte, minoritaria all'inizio del ' 68 , ma poi capace, nel corso dei mesi, di conquistare l'egemonia nel movimento sindacale, innescando quei cambiamenti organizzativi e rivendicativi che sfociarono infine nell'Autunno caldo. La "sinistra sindacale" - così può essere definita quest'area, presente in modo trasversale nelle tre principali confederazioni (Cgil, Cisl, Uil) coincise di fatto con le più importanti e combattive federazioni industriali, ma fu presente anche, e in misura crescente, nei gruppi dirigenti dei territori più industrializzati e dei centri confederali. I suoi esponenti furono i primi a comprendere la necessità, vitale se non si voleva correre il rischio di finire travolti dalla contestazione, di elaborare una nuova cultura sindacale, dalla quale sarebbe nato, proprio durante l'autunno del '69, il cosiddetto «sindacato dei Consigli» ${ }^{13}$.

In estrema sintesi, dunque, l'Autunno caldo scoppiò soprattutto per la volontà, diffusa tra molti operai, di contestare una "società del benessere" che, pur avendo migliorato le condizioni di vita di ampie fasce della popolazione (anche delle stesse classi popolari), era in stridente contrasto con la realtà concreta dei luoghi di lavoro; e si concluse con «la più ampia e 1992.

${ }^{11}$ G. Arrighi-T.H. Hopkins-I. Wallerstein, Antisystemic movements, manifestolibri, Roma

${ }^{12}$ G. Giugni, Sindacato: anni Settanta, «Economia \& lavoro», 1972, n. 1, ora in Id., Idee per il lavoro, a cura di S. Sciarra, Laterza, Roma-Bari 2020, pp. 74-83.

${ }^{13}$ F. Loreto, L' “anima bella” del sindacato. Storia della sinistra sindacale (1960-1980), Ediesse, Roma 2005. 
secca redistribuzione sociale di reddito, di poteri e di diritti mai avvenuta nel corso della storia repubblicana», grazie all'intreccio virtuoso tra la mobilitazione dei lavoratori e la capacità di una parte del sindacato d'imporre all'insieme delle organizzazioni un tragitto di rinnovamento, radicato e profondo ${ }^{14}$.

Il programma della sinistra sindacale prevedeva di allargare e rafforzare i processi di democrazia interna, riducendo le imposizioni da parte della rappresentanza sindacale tradizionale e aumentando il ricorso agli istituti della democrazia diretta (assemblee, inchieste, referendum, consultazioni), per lasciare le decisioni finali nelle mani dei lavoratori stessi, anche di coloro che non erano iscritti ai sindacati. Inoltre, il nuovo progetto mirava a rafforzare l'autonomia dai partiti, non solo tramite regole formali di incompatibilità, ma soprattutto attraverso l'elaborazione di un proprio programma generale, e dunque politico, che partisse dalla fabbrica per tentare di cambiare la società, attraverso riforme radicali e innovative. Infine, occorreva superare una volta per tutte le incomprensioni del passato, per costruire un futuro concreto di unità tra le diverse sigle confederali, intesa non come mera collaborazione ma come vera e propria comunione di intenti $\mathrm{i}^{15}$.

Lo scontro interno al sindacato non fu indolore poiché causò, a tutti i livelli (territoriale, categoriale, confederale), numerose tensioni e molteplici strappi, anche generazionali; inoltre, esso richiese inevitabilmente un tempo adeguato di elaborazione e sedimentazione. Il periodo determinante per la maturazione della nuova linea si ebbe tra l'autunno del 1968 e l'estate del 1969. In quel lasso di tempo si ottennero le prime conquiste significative, a livello aziendale, in tema di ambiente e organizzazione del lavoro, tramite la prima ampia contestazione dei tempi e dei metodi di produzione; inoltre, in numerose imprese iniziarono a diffondersi il diritto di assemblea e i primi delegati (di linea, di squadra, di reparto e di «gruppo operaio omogeneo», come si diceva allora), i quali progressivamente si appropriarono del potere di contrattazione nei luoghi di lavoro, anche perché eletti da tutti i lavoratori, senza esplicite indicazioni sindacali; infine, tra il maggio e il luglio del '69, si tenne la vasta consultazione di base, decisa dalle federazioni dei metalmeccanici (Fim, Fiom e Uilm), che varò la piattaforma contrattuale, il cui capitolo sugli "aumenti salariali uguali per tutti" relegò in minoranza il gruppo dirigente

${ }^{14}$ La citazione è tratta da P. Ferraris, Millenovecentosessantanove, in 1969, «Parolechiave», 1996, n. 18, p. 17. Cfr. anche A. Becchi-A. Sangiovanni, L'Autunno caldo. Cinquant'anni dopo, Donzelli, Roma 2019 e P. Ferrero, 1969: quando gli operai hanno rovesciato il mondo. Sull'attualità dell'Autunno caldo, DeriveApprodi, Roma 2019.

${ }^{15}$ F. Loreto, Potere sindacale, diritti dei lavoratori e contrattazione collettiva in Italia, 1968-1973, «Italia contemporanea», 2015, n. 278, pp. 247-66. Cfr. N. De Amicis, La difficile utopia del possibile. La Federazione lavoratori metalmeccanici nel «decennio operaio» (1968-1984), Ediesse, Roma 2010. 
della Fiom, la più grande delle tre federazioni. La democrazia deliberativa era ormai un fatto compiuto ${ }^{16}$.

Nello stesso tempo, le direzioni confederali non rimasero a guardare: le due vertenze generali, per la riforma delle pensioni (entrata nel vivo il 14 novembre 1968 con il primo sciopero generale unitario dai tempi delle scissioni del 1948 e culminata nell'approvazione della legge Brodolini) e per la cancellazione delle "zone salariali" (che vide la straordinaria partecipazione dei lavoratori del Sud, i quali, in un colpo solo, riuscirono a ottenere un aumento delle retribuzioni del 15-20\%), rappresentarono tappe fondamentali nella costruzione del sindacato come soggetto politico, protagonista tra l'Autunno caldo e i primi anni '70 della "lotta per le riforme" nell'epoca della supplenza sindacale ${ }^{17}$.

\section{L’Autunno caldo e le trasformazioni della società italiana}

La conflittualità che raggiunse il culmine con l'Autunno caldo e si protrasse ben oltre la crisi petrolifera del 1973-74, comportò sicuramente dei problemi per l'andamento dell'economia italiana. Tuttavia, le cause principali del cedimento della crescita straordinaria del miracolo economico sono ascrivibili innanzitutto a fattori di origine internazionale: la golden age, com'è noto, finì in tutto il mondo occidentale con il crollo del sistema dei cambi fissi, dopo l'inconvertibilità del dollaro, stabilita da Richard Nixon nel 1971, seguito dall'inflazione esportata dall'America e poi esplosa con lo shock petrolifero del 1973; inflazione, certo, ulteriormente alimentata dalla rincorsa salariprezzi, con tassi più elevati in Italia, gravati però dalla maggiore dipendenza del paese dalle importazioni di materie prime. In ogni caso, nonostante la dominante stagflazione, con la ricerca di adattamenti ai cicli brevi attraverso le politiche monetarie di stop and go e con le svalutazioni competitive, i tassi di crescita del Pil nel decennio '70 furono mediamente del 3\%, ovvero su livelli che oggi apparirebbero da sogno.

La conflittualità, come da manuale, spinse le imprese all'innovazione tecnologica e organizzativa: si automatizzarono i settori dove la nocività e la gravosità del lavoro erano più accentuate (ad esempio, la lastroferratura e la verniciatura nella produzione automobilistica), avviando un primo ingresso delle tecnologie informatiche che, a loro volta, attraverso lenti processi di

${ }^{16} \mathrm{~F}$. Loreto, La nascita del sindacato dei consigli: la piattaforma contrattuale unitaria dei metalmeccanici nel 1969, in P. Causarano-L. Falossi-P. Giovannini (a cura di), Il 1969 e dintorni cit., pp. 37-46.

${ }^{17}$ M.L. Righi, Gli anni dell'azione diretta (1963-1972), in L. Bertucelli-A. Pepe-M.L. Righi, Storia del sindacato in Italia nel ' $900, \mathrm{IV}$, Il sindacato nella società industriale, Ediesse, Roma 2008, pp. 105-79. 
learning by doing, aprirono la strada ai modelli organizzativi post-fordisti. L'inquadramento unico operai-impiegati creò le premesse contrattuali per l'accorciamento delle distanze gerarchiche, finalizzato all'utilizzo sistematico dell'esperienza operaia. Certo, si può osservare che gli esiti positivi delle rivendicazioni sindacali, nel tentativo di valorizzare la persona garantendo la coesione e la solidarietà fra lavoratori - le modifiche dell'organizzazione del lavoro, l'inquadramento unico, la prevenzione - furono reinterpretati dalle imprese in chiave di coinvolgimento negli obiettivi aziendali, escludendo le più generali finalità collettive. Tuttavia, nel persistente dualismo delle interpretazioni riguardo agli effetti dei nuovi sistemi organizzativi sulla qualità del lavoro operaio, tra chi ne sottolinea le negatività e chi ne vede aspetti di miglioramento, questi ultimi, fatte salve le specificità dei casi, sembrano prevalere, almeno a livello potenziale ${ }^{18}$.

La circolazione di nuovi valori e modelli culturali veicolati dalla mobilitazione studentesca e operaia abbatté vecchi schemi mentali. Ad esempio ridusse il sospetto e la diffidenza con i quali erano stati originariamente accolti al Nord i migranti dal Mezzogiorno. Tra molteplici ostacoli e conflitti intestini, il "femminismo sindacale" riuscì a inserire stabilmente le questioni di genere nell'agenda delle organizzazioni dei lavoratori affermando in modo autonomo, attraverso la fioritura di numerosi coordinamenti femminili, la centralità dei bisogni e dei diritti delle lavoratrici e delle donne in generale ${ }^{19}$. Come non ricordare poi le 150 ore, simbolo della volontà di riscatto ed emancipazione collettiva del mondo del lavoro dai bassi livelli di scolarità, ai quali il bisogno economico e l'insufficiente tutela del diritto allo studio avevano relegato masse di lavoratori manuali ${ }^{20}$ ? $\mathrm{Fu}$ da lì che prese slancio il tema dell'educazione degli adulti e di seguito la riflessione sull'apprendimento continuo, di fondamentale importanza nella realtà odierna, caratterizzata dal passaggio dal "Lavoro" ai "lavori" e dalla rapida evoluzione delle tecnologie che richiedono l'acquisizione di nuove competenze. Dai corsi sperimentali di scuola media per lavoratori, grazie anche a un corpo docente fortemente motivato, sono nate innovazioni che hanno sedimentato attività integrate tra formazione professionale e istruzione scolastica, nonché il sistema dell'Istruzione tecnica superiore (Its) che, pur non avendo raggiunto un'amplissima diffusione, rappresenta oggi una delle esperienze più significative nel rapporto tra scuola e mondo del lavoro.

Della massima importanza, però, è stata soprattutto la grande stagione di riforme degli anni '70: non soltanto lo Statuto dei diritti dei lavoratori e, più

${ }^{18}$ A. Cipriani-A. Gramolati-G. Mari (a cura di), Il lavoro 4.0. La quarta rivoluzione industriale e le trasformazioni delle attività lavorative, Fup, Firenze 2018.

${ }^{19}$ A. Frisone, Femminismo al lavoro. Come le donne hanno cambiato il sindacato in Italia e in Francia (1968-1983), Viella, Roma 2020.

${ }^{20} \mathrm{~F}$. Lauria, Le 150 ore per il diritto allo studio. Analisi, memorie, echi di una straordinaria esperienza sindacale, Edizioni Lavoro, Roma 2011. 
in generale, il rafforzamento dei diritti sociali, ma anche la conquista di nuovi diritti civili. La tutela delle libertà costituzionali nei luoghi di lavoro e la promozione dell'attività sindacale rispondevano alla volontà di mettere fine all'autoritarismo con il quale il padronato aveva colpito i militanti sindacali negli anni '50 e, in misura minore, nel decennio successivo. Molti articoli dello Statuto, infatti, erano puntualmente finalizzati proprio alla proibizione delle pratiche discriminatorie messe in atto nel clima esasperato della guerra fredda.

Le grandi mobilitazioni del 1968 e del 1969 furono però anche alla base dell'esplosione della domanda di riforme che investì l'intera società: per non richiamare che le più importanti, il divorzio, l'interruzione volontaria di gravidanza contro l'aborto clandestino, il nuovo diritto di famiglia, la chiusura dei manicomi. In alcuni territori poi, specie in Emilia-Romagna e in Toscana, grazie alle rivendicazioni sindacali e all'interlocuzione virtuosa con gli enti locali e le neonate regioni, si ottennero appositi fondi per i servizi sociali, finanziati tramite contributi versati dalle imprese, con cui furono varate importanti misure in tema di trasporti, scuole materne e asili nido, tutti rigorosamente pubblici ${ }^{21}$. A chiusura del ciclo riformatore, infine, nella stagione della "solidarietà nazionale" fu approvata la legge sul Servizio sanitario nazionale, che ebbe la spinta precorritrice proprio nelle iniziative sindacali sulla salute e la sicurezza nei luoghi di lavoro e creò un istituto di welfare finalmente universalistico, dando vita a un sistema ancora oggi ai vertici qualitativi a livello mondiale ${ }^{22}$.

L'impasto complesso e a tratti contraddittorio di cattolicesimo sociale, antimperialismo e terzomondismo, pulsioni anticapitalistiche, revival marxisti, spinte libertarie e rivoluzionarie che si mosse nel "secondo biennio rosso", contestando una società ingessata e socialmente iniqua, produsse la diffusione di valori progressisti e solidaristi, che si riflessero da un lato nelle rivendicazioni sindacali, tutte ispirate all'accorciamento delle distanze sociali, a partire dall'egualitarismo salariale e normativo; dall'altro nella spinta alle riforme. Senza la contestazione delle giovani generazioni, l'"epoca dei Consigli" non si sarebbe forse avviata. Ma fu proprio il "sindacato dei Consigli" il massimo protagonista della stagione delle riforme: per smuovere l'Italia e modernizzarne diritti civili, welfare e relazioni contrattuali, fu indispensabile la scossa impressa da un movimento che, in fin dei conti, può essere considerato rivoluzionario.

${ }^{21}$ Sul caso reggiano, una delle esperienze più avanzate di riformismo sociale, cfr. L. Baldissara (a cura di), Tempi di conflitti, tempi di crisi. Contesti e pratiche del conflitto sociale a Reggio Emilia nei "lunghi anni Settanta", l'ancora del Mediterraneo, Napoli-Roma 2008.

${ }^{22}$ C. Giorgi-I. Pavan, Le lotte per la salute in Italia e le premesse della riforma sanitaria. Partiti, sindacati, movimenti e percorsi biografici, 1958-1978, «Studi storici», 60 (2019), n. 2, pp. 417-55. 